import, the precursor was added at the second incubation step. For crosslinking, mitochondria containing accumulated ${ }^{35} \mathrm{~S}$-labelled preproteins were re-isolated through a sucrose cushion $^{5,12}$, washed and incubated with EGS. After quenching of the crosslinker with Tris buffer, the proteins were precipitated with trichloroacetic acid. The samples were either analysed by SDSPAGE or lysed in SDS-containing buffer and immunoprecipitated in Triton $\mathrm{X}$-100-containing buffer ${ }^{12}$. Standard procedures were used for immunoblotting onto nitrocellulose. Bands were detected by the Enhanced Chemiluminescence System (Amersham). Membrane potential was assessed using $\operatorname{DiSC}_{3}(5)$ (Molecular Probes) $)^{15,16}$

Received 24 February; accepted 7 May 1997

1. Schatz, G. \& Dobberstein, B. Common principles of protein translocation across membranes. Science 271, 1519-1526 (1996)

. Pfanner, N. \& Meijer, M. Mitochondrial biogenesis: the Tom and Tim machine. Curr. Biol. 7, R100R103 (1997)

Lill, R. \& Neupert, W. Mechanisms of protein import across the mitochondrial outer membrane. Trends Cell Biol. 6, 56-61 (1996).

4. Bolliger, L., Junne, T., Schatz, G. \& Lithgow, T. Acidic receptor domains on both sides of the oute membrane mediate translocation of precursor proteins into yeast mitochondria. EMBO J. 14, 63186326 (1995).

Hönlinger, A. et al. The mitochondrial receptor complex: Mom22 is essential for cell viability and directly interacts with preproteins. Mol. Cell. Biol. 15, 3382-3389 (1995).

6. Mayer, A., Nargang, F. E., Neupert, W. \& Lill, R. MOM22 is a receptor for mitochondrial targeting sequences and cooperates with MOM19. EMBO J. 14, 4204-4211 (1995).

Gratzer, S. et al. Mas37p, a novel receptor subunit for protein import into mitochondria. J. Cell Biol. 129, 25-34 (1995).

8. Vestweber, D., Brunner, J., Baker, A. \& Schatz, G. A $42 \mathrm{~K}$ outer-membrane protein is a component of the yeast mitochondrial protein import site. Nature 341, 205-209 (1989).

9. Kiebler, M. et al. Identification of a mitochondrial receptor complex required for recognition and membrane insertion of precursor proteins. Nature 348, 610-616 (1990).

10. Alconada, A., Kübrich, M., Moczko, M., Hönlinger, A. \& Pfanner, N. The mitochondrial recepto complex: the small subunit Mom8b/Isp6 supports association of receptors with the general insertion pore and transfer of preproteins. Mol. Cell. Biol. 15, 6196-6205 (1995).

11. Hönlinger, A. et al. Tom7 modulates the dynamics of the mitochondrial outer membrane translocase and plays a pathway-related role in protein import. EMBO J. 15, 2125-2137 (1996)

12. Alconada, A., Gärtner, F., Hönlinger, A., Kübrich, M. \& Pfanner, N. Mitochondrial receptor complex from Neurospora crassa and Saccharomyces cerevisiae. Meth. Enzymol. 260, 263-286 (1995).

13. Steger, H. F. et al. Import of ADP/ATP carrier into mitochondria: two receptors act in parallel. J. Cell Biol. 111, 2353-2363 (1990).

14. Dekker, P. J. T., Müller, H., Rassow, J. \& Pfanner, N. Characterization of the preprotein translocase of the outer mitochondrial membrane by blue native electrophoresis. Biol. Chem. 377, 535-538 (1996).

15. Eilers, M., Oppliger, W. \& Schatz, G. Both ATP and an energized inner membrane are required to import a purified precursor protein into mitochondria. EMBO J. 6, 1073-1077 (1987)

16. Gärtner, F. et al. Mitochondrial import of subunit Va of cytochrome $c$ oxidase characterized with yeas mutants: indpendence from receptors, but requirement for matrix hsp70 translocase function. J. Biol. Chem. 270, 3788-3795 (1995).

17. Pfanner, N., Tropschug, M. \& Neupert, W. Mitochondrial protein import: nucleoside triphosphates are involved in conferring import-competence to precursors. Cell 49, 815-823 (1987).

18. Söllner, T. et al. Mapping of the protein import machinery in the mitochondrial outer membrane by crosslinking of translocation intermediates. Nature 355, 84-87 (1992).

19. Pfaller, R., Pfanner, N. \& Neupert, W. Mitochondrial protein import: bypass of proteinaceous surface receptors can occur with low specificity and efficiency. J. Biol. Chem. 264, 34-39 (1989).

20. Mayer, A., Neupert, W. \& Lill, R. Mitochondrial protein import: reversible binding of the presequence at the trans side of the outer membrane drives partial translocation and unfolding. Cell 80, 127-137 (1995).

1. Huffaker, T. C., Hoyt, M. A. \& Botstein, D. Genetic analysis of the yeast cytoskeleton. Annu. Rev. Genet 21, 259-284 (1987).

22. Kiebler, M. et al. The mitochondrial receptor complex: a central role of MOM22 in mediating preprotein transfer from receptors to the general insertion pore. Cell 74, 483-492 (1993).

23. Haucke, V., Horst, M., Schatz, G. \& Lithgow, T. The Mas20p and Mas70p subunits of the protein import receptor of yeast mitochondria interact via the tetratricopeptide repeat motif in Mas20p: evidence for a single hetero-oligomeric receptor. EMBO J. 15, 1231-1237 (1996).

24. Mihara, K. \& Omura, T. Cytoplasmic chaperones in precursor targeting to mitochondria: the role of MSF and hsp70. Trends Cell Biol. 6, 104-108 (1996).

25. Brundage, L., Fimmel, C. J., Mizushima, S. \& Wickner, W. SecY, SecE, and Band 1 form the membrane-embedded domain of Escherichia coli preprotein translocase. J. Biol. Chem. 267, $4166-$ 4170 (1992).

26. Esnault, Y., Feldheim, G., Blondel, M. O., Schekman, R. \& Képès, F. SSS1 encodes a stabilizing component of the Sec61 subcomplex of the yeast protein translocation apparatus. J. Biol. Chem. $\mathbf{2 6 9}$ 27478-27485 (1994).

27. Rapoport, T. A., Jungnickel, B. \& Kutay, U. Protein transport across the eukaryotic endoplasmi reticulum and bacterial inner membranes. Annu. Rev. Biochem. 65, 271-303 (1996)

28. Tokatlidis, K. et al. Translocation arrest of an intramitochondrial sorting signal next to Timl1 at the inner-membrane import site. Nature 384, 585-588 (1996).

29. Eckerskorn, C., Mewes, W., Goretzki, H. \& Lottspeich, F. A new siliconized-glass fiber as support for protein-chemical analysis of electroblotted proteins. Eur. J. Biochem. 176, 509-519 (1988).

30. Schägger, H., Cramer, W. A. \& von Jagow, G. Analysis of molecular masses and oligomeric states of protein complexes by blue native electrophoresis and isolation of membrane protein complexes by two-dimensional native electrophoresis. Analyt. Biochem. 217, 220-230 (1994).

Acknowledgements. We thank M. Ryan for comments on the manuscript. This work was supported by the Deutsche Forschungsgemeinschaft, Sonderforschungsbereich 388 Freiburg, the Fonds der Chemischen Industrie (N.P.) and a long-term fellowship from the Human Frontier Science Program (P.J.T.D.).

Correspondence and requests for materials should be addressed to N.P. (e-mail: pfanner@ruf.unifrieburg.de). The sequence of Tom5 has been deposited in the Swiss-Prot protein sequence database, accession no. P80967.

\section{X-chromosome-counting mechanisms that determine nematode sex}

\section{Monique Nicoll, Chantal C. Akerib \& Barbara J. Meyer}

Department of Molecular and Cell Biology, University of California at Berkeley, Berkeley, California 94720-3204, USA

Sex is determined in Caenorhabditis elegans by an X-chromosome-counting mechanism that reliably distinguishes the twofold difference in $\mathrm{X}$-chromosome dose between males (1X) and hermaphrodites $(2 \mathrm{X})^{1,2}$. This small quantitative difference is translated into the 'on/off' response of the target gene, xol-1, a switch that specifies the male fate when active and the hermaphrodite fate when inactive ${ }^{3}$. Specific regions of $X$ contain counted signal elements whose combined dose sets the activity of xol-1 (ref. 4). Here we ascribe the dose effects of one region to a discrete, protein-encoding gene, fox-1. We demonstrate that the dosesensitive signal elements on chromosome X control xol-1 through two different molecular mechanisms. One involves the transcriptional repression of $x o l-1$ in XX animals. The other uses the putative RNA-binding protein encoded by fox-1 to reduce the level of $x o l-1$ protein. These two mechanisms of repression act together to ensure the fidelity of the $\mathrm{X}$-chromosome counting process.

The control of sex by the number of $\mathrm{X}$ chromosomes was the first genetic regulatory mechanism to be described ${ }^{5}$. In the eighty-one years since its discovery, the mechanism by which chromosomes are counted to determine sex has been established only for Drosophila ${ }^{6}$. In C. elegans, the xol-1 gene interprets X-chromosome number ${ }^{3,4}$. Thus, the question of how the worm counts its $\mathrm{X}$ chromosomes has been reduced to the problem of identifying the elements that control xol-1 activity and the mechanism by which they do so. Sex-specific regulation of xol-1 is reflected in the tenfold-higher level of xol-1 transcripts in males than in hermaphrodites ${ }^{3}$. Setting xol-1 expression is essential for viability as well as sex determination because $x o l$ 1 also controls X-chromosome dosage compensation ${ }^{7}$. Dosage compensation equalizes $\mathrm{X}$-chromosome expression between the sexes by halving the level of transcripts made from each $\mathrm{X}$ chromosome in hermaphrodites ${ }^{6,8}$. Inappropriate activation of xol-1 in XX animals prevents the reduction of $\mathrm{X}$ expression and thereby causes hermaphrodite-specific lethality ${ }^{3}$. Repression of xol-1 expression in $\mathrm{XO}$ animals causes inappropriate reduction of $\mathrm{X}$ expression and hence male-specific lethality ${ }^{3,7}$

To understand how the X-chromosome signal elements regulate xol-1, we first investigated whether the sex-specific difference in xol1 transcript levels results from transcriptional regulation. The activity of a lac $Z$ reporter gene under the transcriptional control of the xol-1 promoter (Fig. 1g) was assayed in males and hermaphrodites. We observed that XO animals carrying an integrated array ( $y I s 33)$ containing multiple copies of the Pxol-1::lacZ reporter transgene had high $\beta$-galactosidase activity (Fig. 1c) and that array-bearing XX animals had low activity (Fig. 1b), indicating that transcriptional regulation is central to the sex-specific expression of xol-1.

Our previous genetic analysis demonstrated that X-chromosome dose is communicated to xol-1 by elements in at least three distinct chromosomal regions ${ }^{4}$ (Fig. 1a). Duplication of all three regions causes $>99 \%$ XO-specific lethality ${ }^{4}$. This lethality is suppressed by constitutive expression of xol-l. Deletion of one copy of all three regions in XX animals causes lethality and severe dosage compensation defects that are suppressed by $x o l-1$ null mutations ${ }^{4}$. To understand how individual components of the signal regulate $x o l-1$, we asked whether deletions that eliminate $\mathrm{X}$ signal elements affect 
the transcription of the Pxol-1::lacZ reporter gene. XX embryos from hermaphrodites heterozygous for a deletion that removed the signal element(s) in region $1(y D f 19)$ activated transcription of the Pxol-1::lacZ reporter, indicating that region 1 mediates transcriptional control of xol-1 (Fig. 1d). In contrast, XX embryos from hermaphrodites heterozygous for a deletion that eliminated the signal elements in regions 2 and $3(y D f 20)$ failed to activate the reporter (Fig. 1e). This difference in response cannot be explained by a difference in the relative contribution of the individual $\mathrm{X}$ signal elements to the total $\mathrm{X}$ signal, because a twofold increase in the dose of regions 2 and 3 causes greater XO-specific lethality (62\%) than a

a
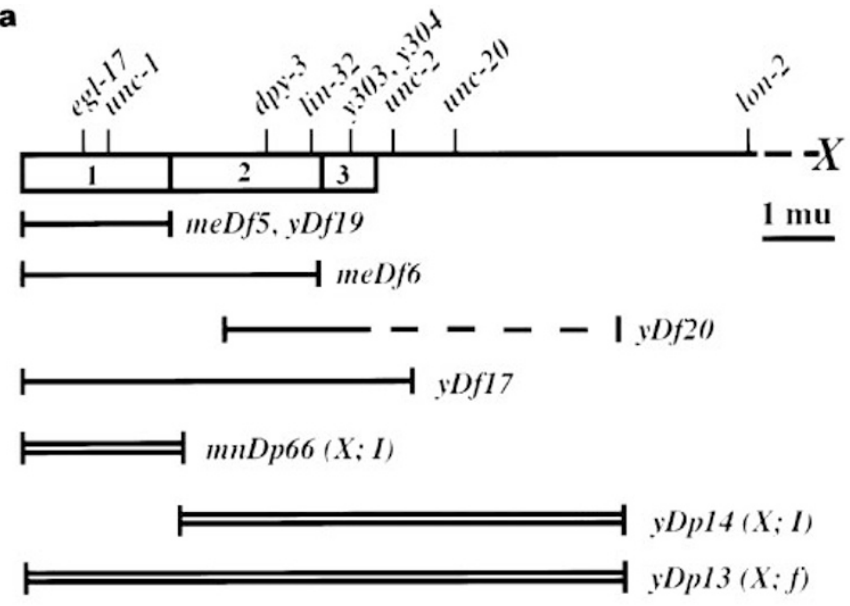

b
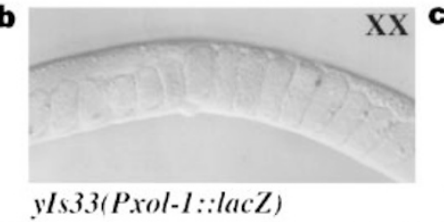

d

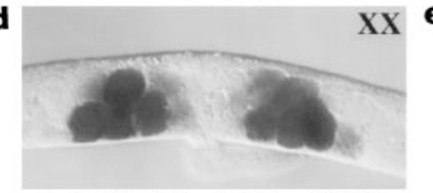

yIs33(Pxol-1::lacZ); yDf19/+

\section{f}

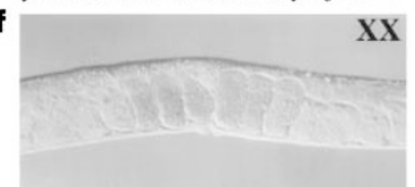

yls33(Pxol-1::lacZ); y303

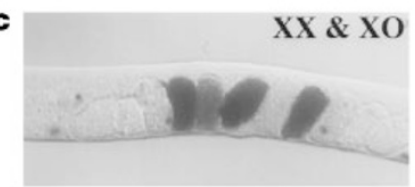

yIs33(Pxol-1::lacZ); him-5

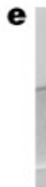

$y I s 33(P x o l-I: \because l a c Z) ; y D f 2() l+$

g

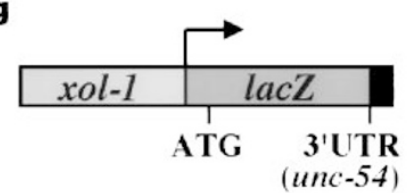

Figure $1 \mathrm{xo} / \mathrm{-1}$ is transcriptionally regulated. a, Genetic map of the X chromosome left end shows three dose-sensitive regions (boxed) that contain counted components of the sex-determination signal. Regional boundaries are defined by deficiency (single line) and duplication (double line) endpoints ${ }^{4}$. b-f. Photomicrographs of embryo-laden midsections of hermaphrodites histochemically stained for $\beta$-galactosidase activity. Each hermaphrodite carries $y / s 33$, an integrated array with multiple copies of the $P x O /-1: . / a c Z$ transcriptional reporter gene $(\mathbf{g})$. This reporter includes $2.8 \mathrm{~kb}$ of $x O /-1$ genomic $5^{\prime}$ regulatory sequences that direct lac Z expression. Comparison of $\beta$-galactosidase activity in $X X$ embryos (b) with that in $X O$ embryos (c) shows the transcriptional regulation of $x O /-1$. The number of embryos showing intense $\beta$-galactosidase expression corresponds to the number of males expected from him-5 mothers, namely $33 \%$ instead of the normal $0.2 \%$. d, XX embryos deficient for region 1 (yDf19) have high $\beta$-galactosidase activity, indicating that signal elements in this region direct the transcriptional repression of $x O /-1$. XX embryos deficient for eitherfox-1 (f) or elements in regions 2 and $3(y D f 20)(\mathbf{e})$ have low $\beta$-galactosidase activity, suggesting that these signal elements are not transcriptional regulators of $x O /-1$.

corresponding increase in the dose of region $1(0 \%)$ (Table 1 , and ref. 4). Because this lethality is suppressed by constitutive expression of $x o l-1$, it is unlikely that the signal elements in regions 2 and 3 regulate a target other than $x o l-1^{4}$. Thus, the expression studies suggest that the $\mathrm{X}$ components of the signal control sex determination and dosage compensation using a combination of two mechanisms, only one of which involves transcriptional repression of xol-1 in XX animals.

To identify specific X-chromosome signal elements and thereby elucidate the mechanism of X-chromosome counting, we conducted a genetic screen for X-linked suppressors of the XO-specific lethality caused by duplication of the three signal element regions within $m n D p 66$ and $y D p 14$ (Fig. 1a, and ref. 4). Based on previous experiments that showed synergism among these signal element regions ${ }^{4}$, we anticipated that loss-of-function mutations in an $\mathrm{X}$ signal element would suppress the male lethality by lowering the total dose of signal elements in the duplication-bearing XO animals. From 24,000 mutagenized X chromosomes, we identified two potential signal-element mutations, $y 303$ and $y 304$, and one deficiency that removes region 1 . Both $y 303$ and $y 304$ were genetically mapped to a small interval covered by $y D p 14$ that corresponds to region 3 (Fig. 1a).

The genetic properties of the $y 303$ and $y 304$ mutations indicate that they define $\mathrm{X}$ signal element(s) in region 3. Both $y 303$ and $y 304$ are dose-dependent suppressors of the $\mathrm{XO}$-specific lethality caused by duplications that increase the number of $\mathrm{X}$ signal elements. The extent of suppression is dependent on the number of wild-type copies of the element identified by $y 303$ and $y 304$. Less than $1 \%$ of duplication-bearing XO animals are viable if they have twice the male dose of the elements in regions 1-3 (either one copy of $y D p 13$ or $m n D p 66 / y D p 14)$ (Table 1 ). In contrast, $100 \%$ of these duplication-bearing $\mathrm{XO}$ animals are viable if they also carry either $y 303$ or y304 (Table 1). Suppression is less effective if more signal elements in region 3 are duplicated. Only $13 \%$ of $y 303$ males and $8.4 \%$ of $y 304$ males are viable if they have two copies of $y D p 14$, a dose of signal elements in regions 2 and 3 (Table 1) that would otherwise kill 100\% of XO animals.

In addition, $y 303$ and $y 304$ cause a synergistic increase in the dosage compensation defects of XX animals that carry deletions of other X signal elements. All XX animals homozygous for either $y 303$ or $y 304$ are viable and appear to be completely wild-type (Fig. 2a,b),

\begin{tabular}{|c|c|c|c|}
\hline Signal elements affected* & Genotype & $\begin{array}{c}\text { Male } \\
\text { viability (\%) }\end{array}$ & $n \dagger$ \\
\hline $\begin{array}{l}D p(1) \text { and } D p(2,3) \ddagger \\
D p(1) \text { and } D p(2,3) \text { and } y 303 \ddagger \\
D p(1) \text { and } D p(2,3) \text { and } y 304 \ddagger\end{array}$ & $\begin{array}{c}m n D p 66 / y D p 14 ;+ \\
m n D p 66 / y D p 14 ; y 303 \\
m n D p 66 / y D p 14 ; y 304\end{array}$ & $\begin{array}{r}1 \\
109 \\
96\end{array}$ & $\begin{array}{r}660 \\
1,031 \\
1,450\end{array}$ \\
\hline $\begin{array}{l}\operatorname{Dp}(1,2,3) \S \\
\operatorname{Dp}(1,2,3) \text { and } y 303 \S \\
\operatorname{Dp}(1,2,3) \text { and } y 304 \$\end{array}$ & $\begin{array}{l}+; y D p 13 \\
y 303 ; y D p 13 \\
y 304 ; y D p 13\end{array}$ & $\begin{array}{l}0.4 \\
90 \\
94\end{array}$ & $\begin{array}{r}266 \\
1,001 \\
747\end{array}$ \\
\hline $\begin{array}{l}\mathrm{Dp}(2,3) \text { and } \mathrm{Dp}(2,3) \| \\
\mathrm{Dp}(2,3) \text { and } \mathrm{Dp}(2,3) \text { and } y 303 \| \\
\operatorname{Dp}(2,3) \text { and } \mathrm{Dp}(2,3) \text { and } y 304 \|\end{array}$ & $\begin{array}{c}y D p 14 / y D p 14 ;+ \\
y D p 14 / y D p 14 ; y 303 \\
y D p 14 / y D p 14 ; y 304\end{array}$ & $\begin{array}{c}0 \\
13 \\
8.4\end{array}$ & $\begin{array}{r}>1,000 \\
507 \\
745\end{array}$ \\
\hline $\begin{array}{l}D p(2,3) \\
D p(1)\end{array}$ & $\begin{array}{c}y D p 14 /+ \\
m n D p 66 /+\end{array}$ & $\begin{array}{r}38 \\
102\end{array}$ & $\begin{array}{r}814 \\
1,874\end{array}$ \\
\hline
\end{tabular}

* Dps are X-chromosome duplications containing one or more of the three signal element regions (Fig. 1a), indicated in parentheses. $y D p 13$ is a free duplication; $y D p 14$ and mnDp66 are attached to chromosomel.

$\uparrow n$, Total number of animals counted.

\$yp 14/+; him-8; unc-2 males were crossed with hermaphrodites of genotype mnDp66; unc-1 dpy-3 or mnDp66; unc-1 dpy-3 y303 or mnDp66; unc-1 dpy-3 y304, and the per cent male viability was calculated as (total non-Dpy non-Unc males/one-half the total non-Dpy male viability was calculated as

non-Unc hermaphrodites $) \times 100$. y303 unc-2 or $d p y-3$ y 304 unc-2, and the per cent male viability was calculated as (total nonDpy non-Unc males/total non-Dpy non-Unc hermaphrodites) $\times 100$.

$\|$ Total live self progeny were counted from several individual hermaphrodites of genotype yDp 14/yDp 14; him-8; unc-2 or yDp 14/yDp 14; him-8; dpy-3 y303 unc-2 or yDp 14/yDp 14; him8: $d p y-3$ y 304 unc-2. Per cent male viability was calculated as (total males)/(0.37 $\times$ total expected progeny) Total expected progeny was calculated as (total hermaphrodites/0.63). Thected progeny). Total expected progeny was calculated as (total hermaphrodites/0.63). These calculations are based on the fact that $37 \%$ of the self progeny of him-8(e1489) hermaphrodites are XO (ref. 14) Data from ref. 4. 
as are $\mathrm{XX}$ animals heterozygous for $m e D f 6$, a deletion that removes the signal elements in regions 1 and 2. However, the combination of lesions in the $y 303 / m e D f 6$ trans-heterozygous XX animals causes pronounced dosage-compensation defects (Fig. 2c). These dosagecompensation defects are comparable to those caused by deletions that remove all three signal element regions, either $y D f 17$ or the heterozygous combination of $y D f 20 / m e D f 5$ (Fig. 2d). This genetic analysis reveals an important role for the locus defined by $y 303$ in counting X chromosomes and suggests that this locus can account for most, if not all of the $\mathrm{X}$ signal activity in region 3 .

y303 and y304 map to a region of X previously identified as causing $\mathrm{XO}$-specific lethality and feminization when present in multiple copies ${ }^{9}$. The genetic locus responsible for the male lethal activity was not defined, but the activity was named fox-1 (for 'feminizing locus on X') and was narrowed to a 30-kilobase (kb) segment of $\mathrm{DNA}^{9}$. A 1.8-kb embryonically expressed complementary DNA from within this segment was predicted to encode a 415amino-acid protein with an RNP motif ${ }^{9}$, suggesting that the protein binds to RNA ${ }^{10}$. DNA sequence analysis of this RNP gene from our mutant strains revealed that the $y 303$ mutation causes an ochre translational stop at codon 37 and is therefore likely to cause complete loss of function (Fig. 2e). The $y 304$ allele causes a proline-to-serine change in the RNP motif at amino acid 161 of the same protein (Fig. 2e). This proline is predicted to be in a small loop region, between the $\beta 1$ sheet and the $\alpha 1$ helix, and is conserved among many of the RNP proteins. This molecular information, together with our genetic analysis of $y 303$ and $y 304$, establishes that the RNP gene, which we designate fox-1, is a bona fide X-chromosome sex-determination signal element. Moreover, because $y 303 \mathrm{XX}$ and $\mathrm{XO}$ animals are fully viable and fertile, and $y 303$ is probably a null allele, fox-1 appears not to be an essential gene.
To learn how the FOX-1 protein functions in the X-chromosome counting process, polyclonal antibodies were raised against the entire 415-amino-acid protein and were used to determine the protein's location in wild-type and fox-1-mutant embryos. We expected a signal element to be expressed by the 28-cell stage and through gastrulation (400-cell stage) because xol-1 must be active during that early period to trigger male development and must be repressed during that period to permit hermaphrodite development ${ }^{3}$. We observed very early nuclear staining in the cells of wild-type XX embryos beginning at the 8-16 cell stage of development; the staining intensity peaked by the 100 -cell stage and was absent by the end of embryonic cell proliferation around the 550-cell stage (Fig. 2f). XX embryos with the fox-1(y303) nonsense mutation lacked staining, establishing the specificity of the antibody and confirming the classification of fox-1(y303) as a null allele (Fig. 2f). The staining results indicate that FOX-1 is present during an appropriate window of time to act as a component of the $\mathrm{X}$ signal and to downregulate $x o l-1$ in hermaphrodites. fox-1 does not seem to regulate the transcription of $x o l-1$. Transcriptional repression of $x o l-1$, as monitored by the $x o l-1:: l a c Z$ transcriptional fusion ( $y I s 33)$, was robust in XX embryos lacking fox-1 (Fig. 1f), as we had found for XX embryos lacking the elements in regions 2 and 3 (Fig. 1e). To demonstrate that FOX-1 nonetheless acts on $x o l-1$ and to address the mechanism of repression, we used a different type of xol-1 reporter gene, one that contained all the xol-1 genomic sequences. In these experiments, the coding region of the green fluorescent protein (GFP) was fused in-frame to the first ATG codon of xol-1, creating a fully bifunctional GFP::XOL-1 fusion protein (Fig. 3d). Eight independent extrachromosomal arrays carrying multiple copies of this $g f p:: x o l-1$ transgene were established in xol-1 mutant strains. In each case, the array suppressed the $\mathrm{XO}$-specific a

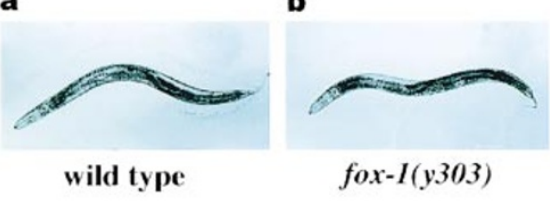

e

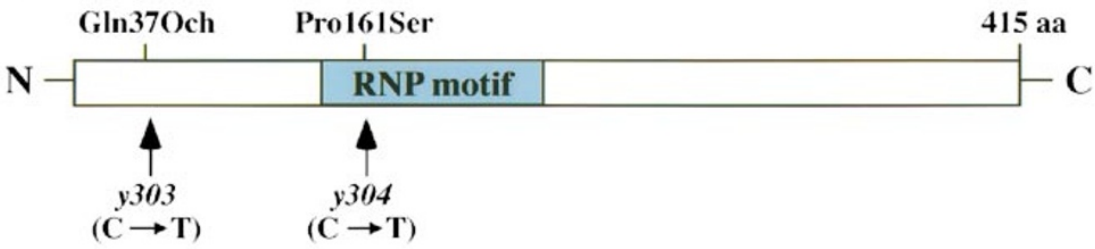

$\mathbf{f}$

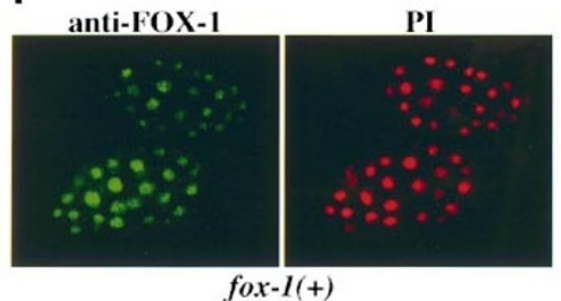

Figure 2 The putative RNA binding protein, FOX-1, is a signal element. a-d, Brightfield photomicrographs comparing wild-type $X X$ animals (a) to $X X$ animals with $X$ chromosome signal element mutations. b, fox-1 mutants appear wild type. c, Loss of one copy of fox-1(+) and one copy of elements in regions 1 and 2 causes synergistic dosage compensation defects (dumpy and egg-laying-defective phenotypes) similar to those caused by loss of one copy of signal elements in c

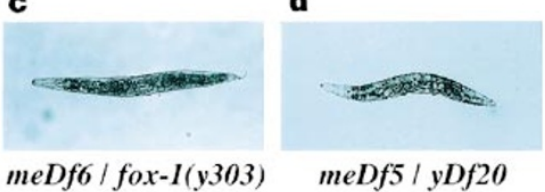


lethality caused by the xol-1 mutation, indicating that the fusion protein has XOL-1 activity. All the arrays expressed high levels of GFP in XO (Fig. 3b) but not XX (Fig. 3a) animals. Moreover, none of these arrays caused XX-specific lethality or dosage compensation defects. Thus, these $g f p:: x o l-1$ reporter arrays showed proper sexspecific regulation. They were crossed into strains that produce high levels of FOX-1 from an integrated array ( $y I s 44)$ containing multiple copies of the fox-1 gene. The level of FOX-1 produced by $y$ Is 44 causes complete $\mathrm{XO}$-specific lethality in otherwise wild-type strains. In $y I s 44$ strains with the $g f p:: x o l-1$ arrays, no GFP expression was detected (Fig. 3c) and all the XO animals died. These results indicate that high doses of FOX-1 drastically reduce the levels of both the GFP::XOL-1 protein and the endogenous XOL-1 protein, revealing that FOX-1 acts through xol-1.

In contrast to the eight $g f p:: x o l-1$ arrays just described, which caused no XX-specific lethality, two other $g f p:: x o l-1$ arrays expressed GFP::XOL-1 at a level that caused complete XX-specific lethality in strains with or without excess FOX-1. These two arrays also suppressed the male-lethal effect of excess FOX-1, suggesting that the FOX-1 activity had been titrated by xol-1 sequences or protein. Such an interpretation is consistent with the fact that xol-1 acts downstream of fox-1, and it suggests the possibility that xol-1 may be the direct target of FOX-1.

Additional evidence for a post-transcriptional role for FOX-1 was provided by a different $x o l-1$ reporter construct in which the $g f p$ coding sequences were fused in frame to a portion of the xol-1 gene containing the promoter region, the 5'UTR, the first two introns and the first 89 codons (Fig. 3h). This fusion, called Pxol-1::gfp, does not provide $x o l-1(+)$ activity. Six independent extrachromosomal arrays carrying multiple copies of this reporter gene cause strong GFP staining in XO (Fig. 3f) but not XX (Fig. 3e) animals,

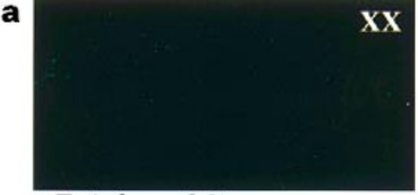
$y E x(g f p:: x o l-1)$

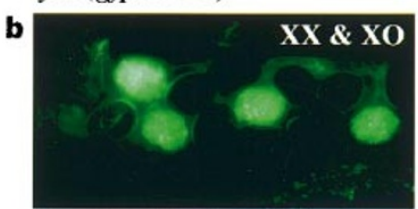

him-5; yEx(gfp::xol-1)

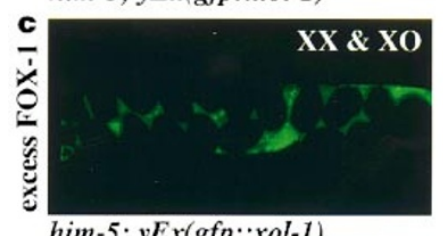

him-5; $y \operatorname{Ex}(g f p:: x o l-1)$

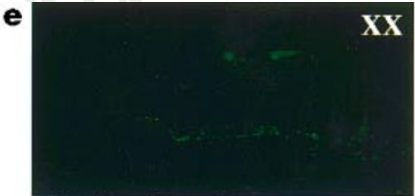
$y \operatorname{Ex}(P x o l-1:: g f p)$

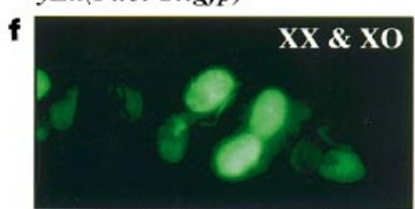

him-5; yEx(Pxol-1::gfp)

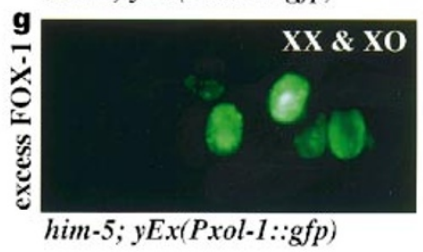

d

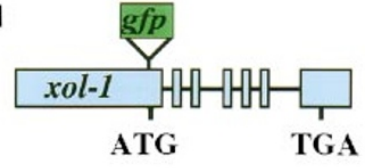

i

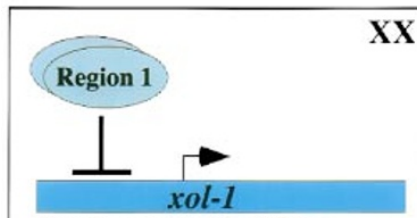

high transcript level

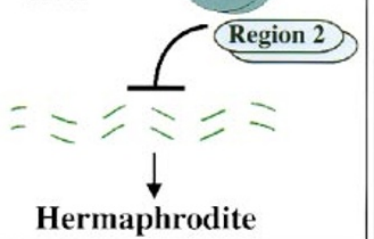

h

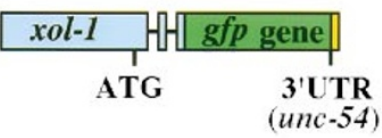

(unc-54)

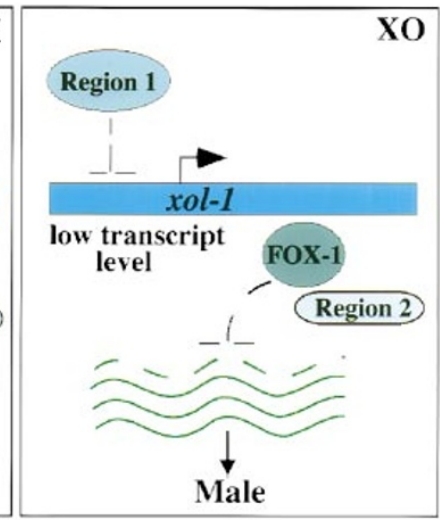

Figure 3 fox-1 represses $x o /-1$. a-c, Fluorescence photomicrographs of embryoladen midsections of hermaphrodites carrying yEx(gfp::xol-1), an extrachromosomal array with multiple copies of the gfp::xol-1 reporter gene in $\mathbf{d}$. In this reporter, the GFP coding sequence was fused in frame to the first ATG of $x O /-1$, thus creating a bifunctional GFP::XOL-1 fusion protein. The sex-specific expression of $y E x(g f p:: x O /-1)$ is shown by the absence of GFP fluorescence in $X X$ embryos (a), and the presence of GFP fluorescence in XO embryos (b). c, Excess FOX-1 provided from an integrated fox-1(+) array, y/s44, represses embryonic GFP fluorescence, showing that FOX-1 acts through $x O /-1$. e-g, Midsections of hermaphrodites carrying yEx(Pxol-1::gfp), an extrachromosomal

array containing multiple copies of the $P x o /-1:: g f p$ translational reporter gene in $\mathbf{h}$. In this reporter, the gfp coding region replaces $x o /-1$ sequences downstream of the 89th codon. e, $\mathbf{f}$, Pxol-1::gfp is sex-specifically expressed. $\mathbf{g}$, Excess FOX-1 fails to repress GFP fluorescence, indicating that $P$ xol-1::gfp lacks FOX-1 target sequences. $\mathbf{i}$, A model for $\mathrm{X}$ chromosome counting by $x 0 /-1$. In XX embryos, $x O /-1$ is inactivated in response to a hermaphrodite dose of $X$-chromoosme signal elements. Complete repression of $\mathrm{xol}-1$ activity involves at least two concerted mechanisms, one acting transcriptionally and a second, mediated by the FOX-1 RNA-binding protein, acting post-transcriptionally. In XO embryos, $x O /-1$ remains active despite the male dose of $\mathrm{X}$-chromosome signal elements. 
switch gene, Sex-lethal, and thereby initiate female development ${ }^{6}$, whereas C. elegans represses expression of its target xol-1 through transcriptional and post-transcriptional mechanisms. No evidence exists for post-transcriptional regulation operating in the initial $\mathrm{X}$ counting process of flies, although they do use a post-transcriptional mechanism to lock in the counting choice ${ }^{6}$. Mammals must also assess their X-chromosome number to achieve dosage compensation through $\mathrm{X}$ inactivation. Although the mechanism has not been determined, the evidence available ${ }^{11}$ implies that it is likely to differ from the chromosome-counting mechanisms in both flies and worms.

\section{Methods}

Genetic mapping. $y 303$ and $y 304$ were isolated using a previously described screen $^{4}$. Of 948 embryos counted from fox-1(y303) XX hermaphrodites, all developed into fertile adults. $y 303$ was mapped between lin-32 and unc-2 by analysing Unc non-Dpy and Dpy non-Unc recombinant progeny from $d p y-3+y 303$ unc-2/+lin-32++mothers. Of 21 Unc non-Dpy recombinants, 4 recombined between $d p y-3$ and lin-32, 5 between lin-32 and $y 303$, and 12 between $y 303$ and $u n c-2$. Of 6 Dpy non-Unc recombinants, 2 recombined between lin-32 and $y 303$ and 4 between $y 303$ and unc-2. y304 was similarly mapped. Of 16 Unc non-Dpy recombinants, 4 recombined between $d p y-3$ and lin-32, 7 between lin-32 and y304, and 5 between $y 304$ and unc-2. Of 17 Dpy non-Unc recombinants, 5 recombined between $\operatorname{lin}-32$ and $y 303$, and 12 between $y 304$ and unc-2. $y D f 19$ is a $\gamma$ ray-induced mutation that fails to complement egl-17 and unc-1 and does not cause X-chromosome nondisjucntion. Of 976 self progeny from $y D f 19 / u n c-1 d p y-3$ mothers, none was male. meDf6 removes lin-32 but probably not fox-1. Fourteen dead embryos from $m e D f 6 / d p y-3$ unc-2 mothers amplified a control band and a fox-1-specific band in single-embryo polymerase chain reactions (PCRs). $y D f 20$ is a suppressor of the XO-specific lethality caused by two copies of $y D p 14$ (I. Carmi and B.J.M., unpublished results) and deletes $d p y-3, \operatorname{lin}-32$ and fox-1. Fifteen dead embryos from $y D f 20 / u n c-1 d p y$-3 mothers that amplified a control band failed to amplify a fox-1-specific band in single-embryo PCR. $y D f 17$ was isolated as a suppressor of the XO-specific lethality caused by $m n D p 66 / y D p 14$. $y D f 17$ removes the left end of $\mathrm{X}$, including $u n c-2$, and $y D f 17 /+\mathrm{XX}$ animals have an Sdc phenotype similar to that of $y D f 14 /+\mathrm{XX}$ animals (ref. 4, and I. Carmi and B.J.M., unpublished results). For single-embryo PCRs ${ }^{12}$, the primer pairs 5'-CCCATTCAGATTCAGAGACC and 5'-CGAGTGAACACGAGCTGTAG amplified an internal fragment of fox-1 (ref. 9). The control primer pairs $5^{\prime}-$ CTACTGTCGACAATGTTGGAATCCTC and 5'-GGGATTTCTGCAGTTGCAAGATG amplified a fragment of $s d c-3$.

DNA sequence analysis of $y 303$ and $y 304$. The DNA changes caused by $y 303$ and $y 304$ were determined by DNA sequence analysis of cDNA generated by reverse-transcribed PCRs of RNA extracted from mutant ( $d p y-3 y 303$ and $d p y-3$ y304) and parental (mnDp66; unc-1 dpy-3) strains. fox-1 was amplified using the primers $5^{\prime}$-CCGCTCGAGATGCAAGCCCTGTACCAACT or 5'CAGTCGGCGTTTGGAATGGATCC with 5'-CGGGATCCCACTCAATACGG AGTAAATCG. The PCR products for each strain were cloned for three independent reactions and were sequenced from three clones. All three sets of sequences from the $y 303$ strain revealed only a C-to-T transition at nucleotide 109, and from the $y 304$ strain, a C-to-T transition at nucleotide 481.

xol-1 reporter genes and transgenic arrays. The Pxol::lac $Z$ transcriptional fusion (pMN21) was made by insertion of the 2,779-bp EcoRI fragment from the xol-1 promoter region into the SmaI site of pPD95.03, an intron-rich version of pPD16.01 (ref. 13) (from A. Fire). The xol-1/lacZ junction occurs $71 \mathrm{bp}$ upstream of the first ATG in $x o l-1$, thus eliminating xol-1's normal 5'UTR, made by the insertion of an SL1 trans-spliced leader 13 nucleotides upstream of that ATG. $y I s 33$ was made by integrating an extrachromosomal array containing pRF4, a plasmid encoding a dominant rol-6(su1006) marker, and pMN21 coinjected at $100: 40 \mu \mathrm{g} \mathrm{ml}^{-1}$. The $g f p$-tagged $x o l-1$ genomic fusion (pMN45) ( $g f p:: x o l-1)$ was made by inserting a PCR fragment of $g f p$ coding sequences (a S65T, I167T variant from Y. Jin) in frame at the first ATG of xol-1. The $y E x(g f p:: x o l-1)$ extrachromosomal arrays contain $\mathrm{p} 76-16 \mathrm{~B}$, a plasmid that encodes $u n c-76(+)$, and pMN45 co-injected at $150: 10 \mu \mathrm{g} \mathrm{ml}^{-1}$. The Pxol-1::gfp translational fusion (pMN15) was made by replacing the 3,441-bp SphI-ApaI fragment from genomic xol-1 sequences with the 1,931-bp SphI-ApaI $g f p$ fragment from the expression vector pPD95.67, which encodes the $565 \mathrm{C}$ variant of GFP (from A. Fire). The $y E x($ Pxol-1::gfp) extrachromosomal arrays contain p76-16B and pMN15 coinjected at 150:20 $\mathrm{g} \mathrm{ml}^{-1}$. More than one thousand embryos of each appropriate genotype were scored in experiments using xol-1 reporter constructs. The XO-specific expression of transgenic arrays carrying these reporter constructs was shown by the lack of embryonic activity in transgenic strains that produced only wild-type XX embryos and the abundant embryonic activity in transgenic lines that produced wild-type XO embryos because they carried a him-5 mutation ${ }^{14}$. Excess fox-1 was produced from an integrated array, $y I s 44$, which causes XO-specific lethality and contains multiple copies of the fox-1-containing cosmid R04B3 and pRF4.

FOX-1 antibodies. Rabbit polyclonal antibodies were raised against a bacterially expressed HIS6::FOX-1 fusion protein that included the entire FOX-1 protein. The fox-1 open reading frame was cloned into the expression vector pRSET-A (Invitrogen), and the HIS6::FOX-1 fusion protein was expressed and purified according to the procedures of Qiagen. Antibodies were affinitypurified using a fusion protein containing the entire FOX-1 protein and the maltose-binding protein (New England Biolabs). Embryos were stained as described $^{15}$ using a 1:50 dilution of affinity-purified FOX-1 antibodies.

Received 2 April; accepted 13 May 1997.

. Nigon, V. Polyploidie experimentale chez un nematode libre, Rhabditis elegans Maupas. Bull. Biol. Fr. Belg. 85, 187-225 (1951)

2. Madl, J. E. \& Herman, R. K. Polyploids and sex determination in Caenorhabditis elegans. Genetics 93, 393-402 (1979)

3. Rhind, N. R., Miller, L. M., Kopczynski, J. B. \& Meyer, B. J. xol-1 acts as an early switch in the C. elegans male/hermaphrodite decision. Cell 80, 71-82 (1995).

4. Akerib, C. C. \& Meyer, B. J. Identification of X chromosome regions in Caenorhabditis elegans that contain sex-determination signal elements. Genetics 138, 1105-1125 (1994).

Bridges, C. B. Non-disjunction as proof of the chromosome theory of heredity. Genetics 1, 1-52 (1916).

6. Cline, T. W. \& Meyer, B. J. Vive la différence: males vs females in flies vs worms. Annu. Rev. Genet. 30, 637-702 (1996).

7. Miller, L. M., Plenefisch, J. D., Casson, L. P. \& Meyer, B. J. xol-1: A gene that controls the male modes of both sex determination and X chromosome dosage compensation in C elegans. Cell 55, 167-183 (1988).

8. Meyer, B. J. \& Casson, L. P. Caenorhabditis elegans compensates for the difference in $X$ chromosome dosage between the sexes by regulating transcript levels. Cell 47, 871-881 (1986).

9. Hodgkin, J., Zellan, J. D. \& Albertson, D. G. Identification of a candidate primary sex determination locus, fox-1 on the X chromosome of Caenorhabditis elegans. Development 120, 3681-3689 (1994).

10. Bird, C. G. \& Dreyfuss, G. Conserved structures and diversity of functions of RNA-binding proteins. Science 265, 615-621 (1994).

11. Willard, H. F. X chromosome inactivation, XIST, and pursuit of the X-inactivation center. Cell 86, 5-7 (1996).

12. Barstead, R. J., Kleiman, L. \& Waterston, R. H. Cloning, sequencing and mapping of an $\alpha$-actinin gene from the nematode Caenorhabditis elegans. Cell Motil. Cytoskel. 20, 69-78 (1991).

13. Fire, A., Harrison, S. W. \& Dixon, D. A modular set of lacZ fusion vectors for studying gene expression in Caenorhabditis elegans. Gene 93, 189-198 (1990).

14. Hodgkin, J., Horvitz, H. R. \& Brenner, S. Nondisjunction mutants of Caenorhabiditis elegans. Genetics 91, 67-94 (1979).

15. Chuang, P.-T., Albertson, D. G. \& Meyer, B. J. DPY-27: A chromosome condensation homolog that regulates $C$. elegans dosage compensation through association with the $X$ chromosome. Cell 79, 459474 (1994).

Acknowledgements. We thank I. Carmi, T. Cline, J. Rine, H. Dawes for discussions and critical comments on the manuscript, A. Fire for reporter gene vectors and Y. Jin for the $g f p$ coding sequences. M.N. is a National Science Foundation predoctoral fellow. C.C.A. was an American Cancer Society postdoctoral fellow. This work was supported by a grant from the National Institutes of Health to B.J.M.

Correspondence and requests for materials should be addressed to B.J.M. (e-mail: meyer@mendel. berkeley.edu)

\section{correction}

\section{Structure of the adenylyl cyclase catalytic core}

\section{Gongyi Zhang, Yu Liu, Arnold E. Ruoho \& James H. Hurley}

Nature 386, 247-253 (1997)

In Fig. 3, the sequence in line 2 from 908 to 943 should read PGELVHMLNELFGKFDQIAKENEC...MRIKILGDC, and from 1,004 to 1,022 should be NVLCGVIGLQKWQYDVWSH. An extraneous 19 amino acids appeared at the start of the third line of the last block of sequence. This line should therefore start with AGGRA.... Also, the correct GenBank accession code for bovine type 1 adenylyl cyclase is M25579. 